\title{
EGFR-mutant Non-small Cell Lung Cancer Accompanied by Transient Asymptomatic Pulmonary Opacities Successfully Treated with "Stop-And-Go" Osimertinib
}

\author{
Keigo Kobayashi ${ }^{1}$, Katsuhiko Naoki ${ }^{1,2}$, Aoi Kuroda ${ }^{1}$, Hiroyuki Yasuda ${ }^{1}$, Ichiro Kawada ${ }^{1}$, \\ Kenzo Soejima ${ }^{1,3}$ and Tomoko Betsuyaku ${ }^{1}$
}

\begin{abstract}
:
A 69-year-old man with post-operative recurrence of lung adenocarcinoma was treated with multiple chemotherapies, including epidermal growth factor receptor (EGFR)-tyrosine kinase inhibitors. A second biopsy revealed an EGFR T790M mutation. As 10th-line chemotherapy, osimertinib was initiated. After 24 weeks, chest computed tomography (CT) revealed asymptomatic ground-glass opacities in both lobes. After four weeks of osimertinib discontinuation, imaging revealed rapid lung cancer progression. Osimertinib was resumed. After 11 weeks, CT revealed decreased lung nodules with no exacerbation of interstitial lung disease. We describe a patient who experienced transient asymptomatic pulmonary opacities during treatment with osimertinib, which was successfully managed by a "stop-and-go" approach.
\end{abstract}

Key words: non-small cell lung cancer, osimertinib, drug-induced pneumonitis, stop-and-go, EGFR-T790M, transient asymptomatic pulmonary opacities

(Intern Med 57: 1007-1010, 2018)

(DOI: 10.2169/internalmedicine.9609-17)

\section{Introduction}

Osimertinib has been approved as the standard treatment for epidermal growth factor receptor (EGFR) mutationpositive non-small cell lung cancer (NSCLC) that is resistant to first- and second-generation EGFR tyrosine kinase inhibitors (TKIs) owing to the acquisition of the T790M mutation $(1,2)$. The incidence of osimertinib-induced interstitial lung disease (ILD) was reported as $4 \%$, and death was observed in $1 \%$ of cases (1). Although ILD is a serious adverse drug reaction of EGFR-TKIs $(3,4)$, there is no strong evidence supporting any TKI-induced ILD treatment. The current management of ILD includes oxygen inhalation, drug discontinuation, and high-dose corticosteroids or immunosuppressants (4). Noonan et al. recently reported that frequent transient asymptomatic pulmonary opacity (TAPO) occurred in $35 \%$ of patients (7/20) during osimertinib treatment (5). Based on their findings, they suggested that it might be reasonable to continue treatment with osimertinib if new-onset pulmonary lesions are asymptomatic and localized.

We herein report a patient who experienced TAPO during treatment with osimertinib and was successfully managed by stopping and rechallenging ("stop-and-go") with osimertinib.

\section{Case Report}

A 69-year-old man was diagnosed with stage IB (T2NOM0) lung adenocarcinoma. He underwent left upper lobectomy. Thirty-five months after surgery, computed tomography (CT) revealed multiple lung metastases. Molecular profiling revealed that the tumor harbored the EGFR L858R mutation. Thereafter, multiple lines of chemotherapy with different agents, including EGFR-TKIs (gefitinib, erlotinib, and afatinib) and cytotoxic agents, were administered. During these treatment periods, a second biopsy was performed, and an additional EGFR T790M mutation was

${ }^{1}$ Division of Pulmonary Medicine, Department of Medicine, Keio University School of Medicine, Japan, ${ }^{2}$ Cancer Center, Keio University School of Medicine, Japan and ${ }^{3}$ Clinical and Translational Research Center, Keio University School of Medicine, Japan Received: June 5, 2017; Accepted: August 17, 2017; Advance Publication by J-STAGE: December 21, 2017 Correspondence to Dr. Katsuhiko Naoki, knaoki@pg7.so-net.ne.jp 
(A)

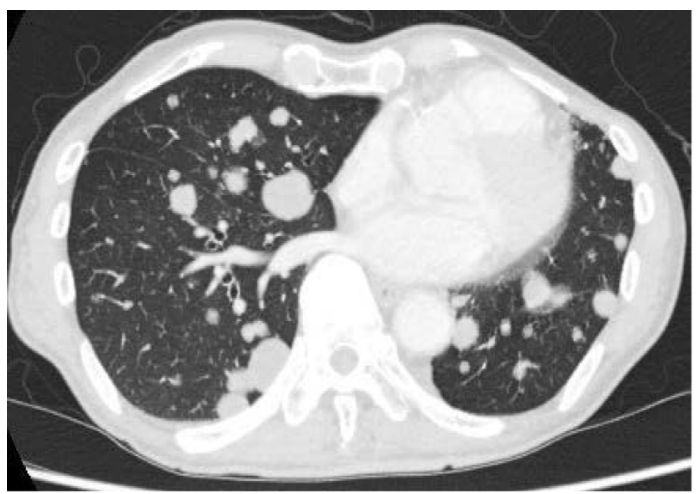

(C)

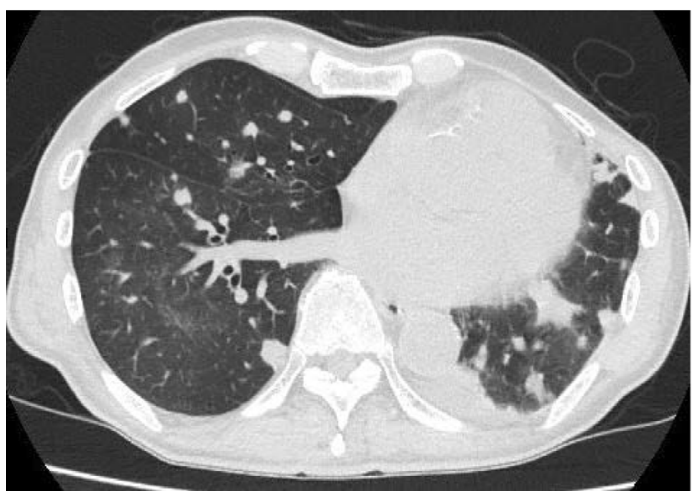

(B)

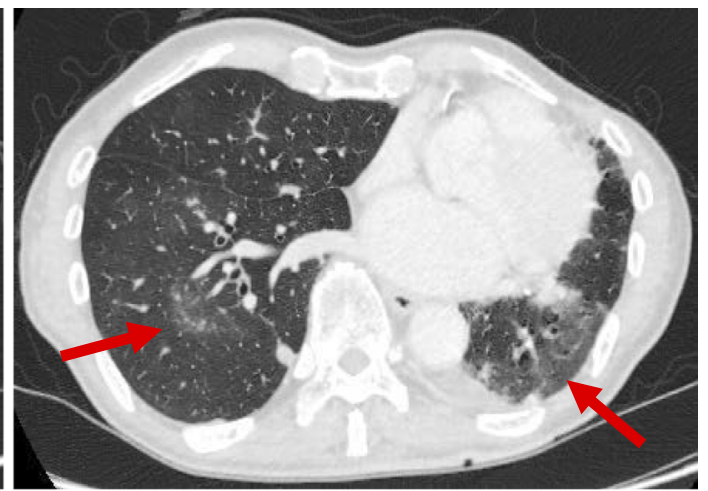

(D)

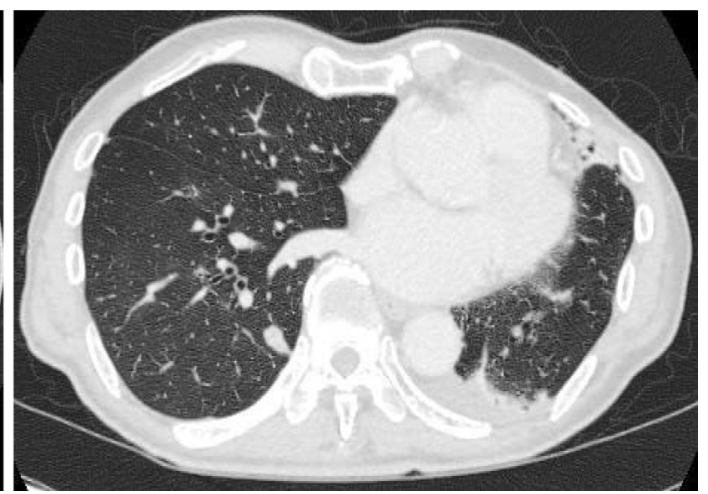

Figure 1. CT scans of the chest (A) before osimertinib treatment, (B) after 24 weeks of osimertinib treatment, (C) after 4 weeks of discontinuation of osimertinib, and (D) after 11 weeks of reinitiation of osimertinib.

confirmed.

As 10th-line chemotherapy, osimertinib ( $80 \mathrm{mg} / \mathrm{day})$ was initiated on June 2016 (Fig. 1A). Eight weeks after starting osimertinib, whole-body CT and magnetic resonance imaging of the brain were performed, showing drastic shrinkage of the lung and brain metastasis. After 24 weeks of treatment, although the systemic response had continued, chest CT revealed asymptomatic ground-glass opacities (GGOs) in both lower lobes (Fig. 1B). Osimertinib was stopped because this development was regarded as osimertinib-induced ILD. We were certain that this was not a pulmonary infection but rather another disease, as his clinical symptoms were not typical of an infectious disease and his C-reactive protein levels were negative. Therefore, we did not conduct any further infection surveys, such as bronchoalveolar lavage.

After four weeks of osimertinib discontinuation, imaging revealed rapid lung cancer progression with multiple novel nodules in both lungs, but the GGOs had resolved slightly (Fig. 1C). Of note, Noonan et al. reported that it might be reasonable to continue treatment with osimertinib in situations of "TAPO" during osimertinib therapy (5). Given this suggestion, and because the patient had no oxygen desaturation, osimertinib treatment was resumed after discussing the approach with the patient. While we suspected he had an osimertinib-induced ILD, the radiological pattern did not re- flect diffuse alveolar damage (DAD), which is known to be a lethal pattern. We therefore reinitiated osimertinib without steroid therapy (6).

After four weeks of osimertinib reinitiation, chest radiograph revealed shrinkage of the multiple lung nodules. In addition, his serum Krebs von den Lungen-6 (KL-6) levels had decreased markedly from $911 \mathrm{U} / \mathrm{mL}$ to $664 \mathrm{U} / \mathrm{mL}$ over 4 weeks (Fig. 2). After 11 weeks of osimertinib reinitiation, whole-body CT revealed decreased multiple lung nodules with no exacerbation of interstitial reticular marking (Fig. 1D)

\section{Discussion}

To our knowledge, this is the first case report of NSCLC with TAPO successfully managed with discontinuation and rechallenge ("stop-and-go") with osimertinib.

Although osimertinib is effective against both EGFRsensitizing and resistant (T790M) mutations, the incidence of osimertinib-induced ILD was reported as $4 \%$, and death was observed in $1 \%$ of cases (1). There is no standard treatment for osimertinib-induced ILD. The general principle of management therefore includes oxygen support, drug discontinuation, and the administration of corticosteroids or immunosuppressants. One case of osimertinib-induced ILD was successfully managed with corticosteroids and a dose 


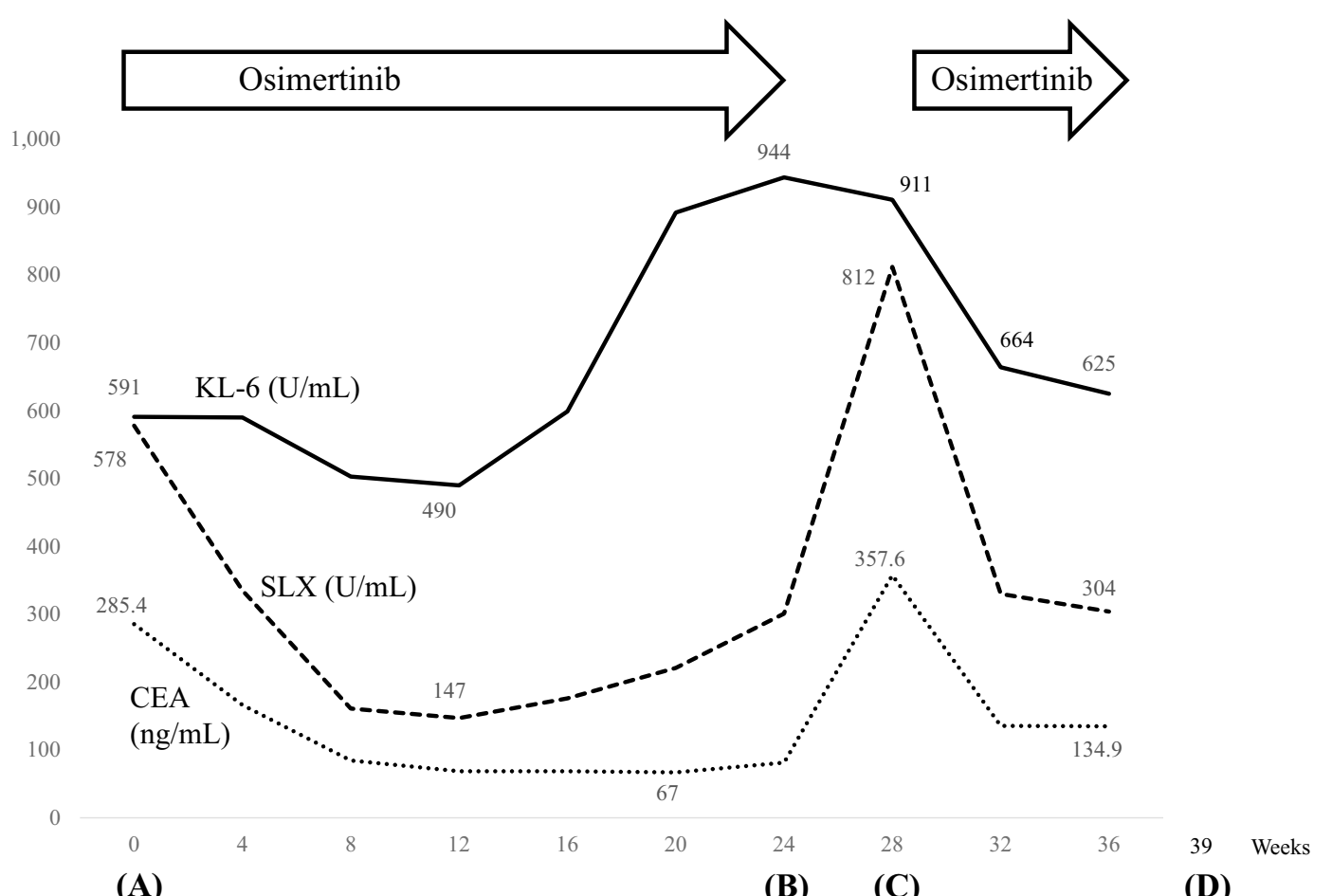

(A)

(B)

(C)

(D)

Figure 2. The vertical axis shows the values of Krebs von den Lungen-6 (KL-6), sialyl Lewis X-i antigen (SLX), and carcinoembryonic antigen (CEA), and the horizontal axis shows the number of weeks from osimertinib initiation. Figure correspondence: 8 weeks on Fig. 2 to Fig. 1 (A), 24 weeks on Fig. 2 to Fig. 1 (B), 28 weeks on Fig. 2 to Fig. 1 (C), and 39 weeks on Fig. 2 to Fig. 1 (D).

reduction of osimertinib (7).

Noonan et al. reported that TAPO occurred during osimertinib treatment in $35 \%(n=7 / 20)$ of patients, and the radiological patterns included GGO with or without nodular consolidation (5). The median time to develop GGO was 8.7 (range, 1.6-43) weeks, with a median time to resolution of 6 (range, 1-11) weeks. In the present case, multiple localized asymptomatic GGOs developed after 24 weeks of osimertinib treatment. Although the patient had normal oxygen saturation levels, we believed the GGO to be indicative of osimertinib-induced ILD and therefore decided to discontinue the drug. After four weeks of discontinuation of osimertinib, the pulmonary lesions improved, although not completely; however, after outweighing the risk of osimertinib-induced ILD, we reinitiated the drug treatment because of the rapid lung cancer progression. After 11 weeks of reinitiation of osimertinib, the multiple lung metastases had shrunk remarkably, and the GGOs had improved.

Generally, physicians tend to discontinue osimertinib treatment when new-onset pulmonary lesions associated with GGO occur, which is reasonable, as the shadows can sometimes indicate fatal drug-induced ILD. However, as osimertinib is the only highly effective drug for treating EGFRT790M-mutation positive NSCLC at present, it might be useful to rechallenge with osimertinib if new pulmonary opacities are not worsened during short-term rest or discontinuation with careful monitoring of the patient's symptoms and radiological findings, because it may be TAPO. We recommend a drug absence of at most 6 weeks, which is the median time for TAPO resolution with careful monitoring, because we are always apprehensive about disease flare. If the shadow changes to any other radiological pattern, such as a DAD pattern, high-dose corticosteroids should be administered.

The "stop and go" approach is a strategy used to avoid treatment-related adverse events as in the management of colorectal cancer with oxaliplatin (8). This practical "stopand-go" approach may be a better, safer, and more reasonable management strategy for new-onset pulmonary lesions occurring during osimertinib treatment than "continuation" management. Further studies are required to investigate the currently unknown mechanisms underlying TAPO. In addition, cases of TAPO alone should be gathered in order to elucidate the radiological and/or clinical factors that distinguish TAPO from osimertinib-induced severe ILD.

\section{Conclusion}

During treatment with osimertinib, carful observation is needed for the early detection of drug-induced ILD. However, because new shadows might be TAPO, the "stop-andgo" administration of osimertinib with careful monitoring may be a reasonable and safe treatment strategy for nonmetastatic new pulmonary shadows occurring during osimertinib treatment, if the pulmonary lesions are asymptomatic and localized.

The authors state that they have no Conflict of Interest (COI). 


\section{References}

1. Yang JC, Ahn MJ, Kim DW, et al. Osimertinib in pretreated T790 M-positive advanced non-small-cell lung cancer: AURA study phase II extension component. J Clin Oncol 35: 1288-1296, 2017.

2. Janne PA, Yang JC, Kim DW, et al. AZD9291 in EGFR inhibitorresistant non-small-cell lung cancer. N Engl J Med 372: 16891699, 2015

3. Inoue A, Saijo Y, Maemondo M, et al. Severe acute interstitial pneumonia and gefitinib. Lancet 361: 137-139, 2003.

4. Tani T, Naoki K, Asakura T, et al. Successful treatment of nonsmall-cell lung cancer with afatinib and a glucocorticoid following gefitinib- and erlotinib-induced interstitial lung disease: A case report. Mol Clin Oncol 5: 488-490, 2016.

5. Noonan SA, Sachs PB, Camidge DR, et al. Transient asympto- matic pulmonary opacities occurring during osimertinib treatment. J Thorac Oncol 11: 2253-2258, 2016.

6. Endo M, Johkoh T, Kimura K, Yamamoto N. Imaging of gefitinibrelated interstitial lung disease: multi-institutional analysis by the West Japan Thoracic Oncology Group. Lung Cancer 52: 135-140, 2006.

7. Nie KK, Zou X, Geng CX, et al. AZD9291-induced acute interstitial lung disease. Chin Med J (Engl) 129: 1507-1508, 2016.

8. Hochster HS. Stop and go: yes or no? J Clin Oncol 27: 56775679, 2009.

The Internal Medicine is an Open Access article distributed under the Creative Commons Attribution-NonCommercial-NoDerivatives 4.0 International License. To view the details of this license, please visit (https://creativecommons.org/licenses/ by-nc-nd/4.0/).

(C) 2018 The Japanese Society of Internal Medicine Intern Med 57: 1007-1010, 2018 